\title{
A Review on the Potential of Bruton's Tyrosine Kinase (Btk) Inhibitor - Ibrutinib for Treatment of Multiple Myeloma (MM)
}

\author{
Sze-Ting Bong ${ }^{1 *}$, Lydia Ngiik-Shiew Law², Jodi Woan-Fei Law ${ }^{3}$ \\ ${ }^{1}$ Department of Biochemistry and Molecular Biology, Bio21 Molecular Science and Biotechnology Institute, University of \\ Melbourne, Parkville, Victoria, Australia \\ ${ }^{2}$ Monash Credentialed Pharmacy Clinical Educator, Faculty of Pharmacy and Pharmaceutical Sciences Monash University, \\ 381 Royal Parade, Parkville VIC 3052 \\ ${ }^{3}$ Novel Bacteria and Drug Discovery (NBDD) Research Group, Microbiome and Bioresource Research Strength, Jeffrey Che- \\ ah School of Medicine and Health Sciences, Monash University Malaysia, Bandar Sunway, Selangor Darul Ehsan, Malaysia
}

\begin{abstract}
Multiple myeloma (MM) is characterized by the over-production of monoclonal plasma cells that eventually become malignant in the bone marrow. MM remains as an incurable cancer, but it can be treated through chemotherapy. Nonetheless, research on novel therapies for effective treatment of MM is ongoing and in this case the involvement of Bruton's tyrosine kinase (Btk) in B cell malignancies has made it one of the new therapeutic targets. In MM patients, it has been reported that the expression of Btk was elevated and this could potentially contribute to chemoresistance indirectly via enhancement of cell proliferation and survival. Ibrutinib is a highly selective irreversible Btk inhibitor commonly used as treatment for B cell malignancies such as Mantle Cell Lymphoma (MCL) and Chronic Lymphocytic Leukemia (CLL). With reference to the potential involvement of Btk in MM and current treatment of MCL and CLL using ibrutinib, researchers have begun to examine the effect of ibrutinib treatment on MM. This review provides information on the association of MM and Btk in conjunction with the treatment using ibrutinib. To date, clinical trials of ibrutinib as therapeutic alternative for MM have produced promising results, particularly as combination therapy with other agents such as dexamethasone and carfilzomib. However, there is limited evidence on the Btk mechanisms involved in MM, hence, it is important to further investigate the Btk oncogenic signalling pathway(s) in MM cells in order to establish successful and improved treatment of MM.
\end{abstract}

Keywords: Multiple myeloma; ibrutinib; cancer; blood; chemotherapy

Received: $15^{\text {th }}$ May 2019

Accepted: $22^{\text {nd }}$ June 2019

Published Online: $30^{\text {th }}$ May 2019
*Correspondence to: Sze-Ting Bong, Department of Biochemistry and Molecular Biology, Bio21 Molecular Science and Biotechnology Institute, University of Melbourne, Parkville, Victoria, Australia; bong-123@ hotmail.com.

Citation: Bong ST, Law LNS, Law JWF. A Review on the Potential of Bruton's Tyrosine Kinase (Btk) Inhibitor - Ibrutinib for Treatment of Multiple Myeloma (MM). Prog Microbes Mol Biol 2019; 2(1): a0000028.

\section{INTRODUCTION}

Multiple myeloma (MM) or myeloma is a B cell malignancy initiated from the over-production and accumulation of malignant plasma cells in the bone marrow ${ }^{[1]}$. It is a progressive disease with increasing occurrence in elderly group. Among the different types of blood cancers, $15 \%$ consisted of the disorder of bone marrow $\mathrm{MM}^{[2]}$. In $\mathrm{MM}$, the over-proliferative malignant plasma cells outcompete the population of other blood cells in bone marrow which can cause some of the disease symptoms such as anaemia. As antibody-producing cells, the malignant plasma cells produce large quantities of a monoclonal antibody (also called $\mathrm{M}$ protein) in $\mathrm{MM}^{[3]}$. Excessive $\mathrm{M}$ protein will interfere with the normal function of immune system. The protein could be accumulated in the kidney, thereby causing renal failure and patients become prone to infections ${ }^{[3]}$. Also, the malignant plasma cells could over-activate osteoclasts but not osteoblasts in the bone marrow $^{[4]}$. Osteoclast is a type of bone cells responsible for bone resorption, while osteoblast directs bone formation. The over-activated osteoclasts could cause excessive bone resorption, resulting in bone lesion commonly encountered in MM patients ${ }^{[3]}$. Hence, patients often suffer from back pain. Moreover, the continuous release of calcium from bone resorption can cause hypercalcemia (excess calcium level in blood) and further contribute to kidney damage ${ }^{[4]}$.

Presently, treatments such as stem cell transplantation and chemotherapies involving drugs such as thalidomide, bortezomib, dexamethasone and lenalinomide are currently adopted for $\mathrm{MM}^{[5-8]}$. However, most of the drugs did not produce promising results as the complete 
remission only last for a few months to a few years ${ }^{[3,4,9,10]}$. Therefore, new treatment strategies are required for effective treatment of MM. As such, the search for new cell surface or molecular targets has been the aim of many researches to explore additional therapeutic options.

\section{BRUTON'S TYROSINE KINASE (BTK) AS NOVEL TARGET}

\section{The origin, structure and functions of Btk}

In recent years, a non-receptor tyrosine kinase known as Bruton's tyrosine kinase (Btk) has been one of the most discussed promising novel targets that could play a role in the treatment of MM due to its involvement in many $\mathrm{B}$ cell malignancies ${ }^{[11,12]}$. Btk is a predominant cytoplasmic protein consisting of 659 amino acid residues, which mainly expressed in the B cell lineage $\mathrm{e}^{[13-15]}$. It is a member of Tec family protein kinases, a subfamily of nonreceptor tyrosine kinases. Other members of the family include Tec, Itk, Bmx and Txk ${ }^{[16]}$. Tec family kinases are structurally conserved, consisting of Plekstrin Homology $(\mathrm{PH})$ and Tec Homology $(\mathrm{TH})$ domains at the N terminal region, followed by Src Homology 3 (SH3), Src Homology 2 (SH2), and kinase domains (Figure 1) ${ }^{[17]}$.

1

Figure 1. Illustration of Btk's protein linear structure. The full-length protein consists of 659 amino acid residues, with molecular weight of about $76.3 \mathrm{kDa}$. The labelled boxes represent the corresponding domains of Btk. The auto-phosphorylation (Y223) and transphosphorylation (Y551) sites are the important activating phosphorylation sites of Btk (Image adapted from Mohamed et al. (2009) $\left.{ }^{[17]}\right)$.

The Btk has acquired its name in the honor of Dr. Bruton C. Ogden, a paediatrician, who described an immunodeficiency condition known as agammaglobulinemia in year $1952^{[18]}$. This condition was observed in a boy who was first admitted to the clinic at the age of 4.5 years old. Prior to admission, the boy had encountered several infections including the development of recurrent pneumococcal sepsis. Several attempts have been done to determine the cause of the patient's recurrent infections. Finally, Dr. Bruton had achieved a breakthrough in this research - he discovered a complete absence of gamma globulins in the patient's blood sample and thus the patient seemed to be unable to produce any antibodies to combat the infection ${ }^{[18,19]}$. Few years later, studies revealed that the lack of antibody-producing cells is the reason of this observed humoral phenotype. The Xlinked agammaglobulinemia (XLA) is the most common agammaglobulinemias known; lost-of-function mutations had contributed to this inheritable XLA, for which this disorder is associated with Bruton's name, hence, it is also known as Bruton's agammaglobulinemia ${ }^{[19,20]}$. The defects of BTK gene (encoding Btk) located at chromosome $\mathrm{X}$ long arm, Xq. 21.3-22 is the cause of human XLA (X-linked recessive disease) ${ }^{[15,19,21,22]}$. The Btk plays critical roles in mediating signals from cell membrane to transcriptional events in the nucleus, regulating B cell development and/or maturation ${ }^{[20,22]}$. Therefore, the mutation of Btk gene will result in dysfunction of Btk or lack of expression of Btk which could prevent the transition of pre-B cell to mature B cell. Consequently, there will be insufficient number of mature $B$ cells to respond and produce antibodies or immunoglobulins to fight against the pathogenic bacterial infections ${ }^{[20]}$.

Biochemical studies had revealed that PH domain is crucial for Btk to be recruited to plasma membrane by binding to phosphatidylinositol-3, 4, 5triphosphate $\left(\mathrm{PIP}_{3}\right)^{[23-25]}$. This allows Btk to be activated by Src family kinases (SFKs) and subsequently mediate transmission of signals to the downstream substrates ${ }^{[26]}$. Mutations at $\mathrm{PH}$ domain that abolished the binding ability of Btk to $\mathrm{PIP}_{3}$ result in development of X-linked agammaglobulinemia (XLA), indicating the importance of Btk being recruited to plasma membrane for activation ${ }^{[27]}$. Other than that, $\mathrm{PH}$ domain was shown to interact with other proteins like protein kinase $\mathrm{C}$ (PKC), heterotrimeric $\mathrm{G}$ protein subunits, transcription factor (TFII-I, also known as BAP-135) $)^{[28-31]}$. Btk also binds Fas via its PH and kinase domains to prevent interaction between Fas and Fas-associated death domain (FADD), and inhibit Fas-mediated apoptosis ${ }^{[32]}$.

TH domain next to $\mathrm{PH}$ domain is another characteristic structural domain of Tec family kinases. It is made up of a Btk motif (Zinc finger motif) and a proline-rich region $(\mathrm{PRR})^{[33]}$. The Btk motif contains one histidine and three cysteines that are critical for the binding of zinc ions and stabilize the protein. Mutations of the conserved cysteine residues affect protein folding and stability, and hence, XLA is developed ${ }^{[34]}$. The PRR plays role in protein-protein interaction by binding to $\mathrm{SH} 3$ domain. It also binds to Btk itself at SH3 domain, regulating its activity either by preventing its interaction with other cellular proteins or phosphorylates Tyr223 to activate Btk kinase activity ${ }^{[35,36]}$. Besides that, the binding of Btk's SH3 domain to Liar was reported to be important for its nucleocytoplasmic shuttling ${ }^{[37]}$. Similar to the $\mathrm{SH} 2$ domain of other protein tyrosine kinase, it binds to specific tyrosine containing substrate. It was shown to preferentially bind phosphopeptides with the sequence of pYEEI ${ }^{[38]}$. SH2 is important for Btk to interact with Spleen tyrosine kinase (Syk) and B cell linker (BLNK; a protein adaptor), and these interactions were essential for activation of Phospholipase C (PLC $\gamma)$ by Btk ${ }^{[39,40]}$.

In the protein structure of Btk, there are two phosphorylation sites that regulate Btk's kinase activity: Tyr223 in SH3 domain and Tyr551 at the catalytic loop in the kinase domain. Transphosphorylation at Tyr551 increases the kinase activity of Btk and permits further autophosphorylation on Tyr223 for full activation ${ }^{[41-43]}$. Biophysical analysis revealed that Btk adopted an elongated conformation in solution, with minimal interactions among the functional domains ${ }^{[44]}$. SFKs, another subfamily of nonreceptor tyrosine kinase that share the same arrangement of SH3, SH2 and kinase domains in their primary structures as Btk. SFKs are inhibited when their SH3 domain binds the linker between $\mathrm{SH} 2$ and kinase domains, and the SH2 domain binds their C-terminal tail at phosphotyrosine 527 to form a globular inactive conformation ${ }^{[45]}$. In contrast, the elongated structure adopted by Btk suggests that the activity of Btk is regulated by intermolecular interactions between Btk and other cellular proteins 
and/or another Btk molecule instead of forming a globular inactive conformation ${ }^{[17,46]}$. Moreover, deletion of other domains did not affect Btk kinase activity, further confirming that intramolecular interactions among the functional domains are not involved in regulating its kinase activity ${ }^{[47]}$. Nevertheless, the regulatory mechanism remains unclear.

\section{Btk's central role in $\mathrm{B}$-cell receptor $(\mathrm{BCR})$ signal- ling pathways}

$\mathrm{B}$ cell is a type of white blood cells essential for humoral immune response ${ }^{[48]}$. B cell develops from pro-B cell in bone marrow up to mature $\mathrm{B}$ cell which then migrate into periphery tissue. Upon antigen activation, these mature $B$ cells differentiate into antibody-producing plasma cells or memory B cells. Loss-of-function mutations in Btk block the transition from pro-B cell to pre-B cell which causes defect in XLA (Figure 2) ${ }^{[15,22,49]}$.

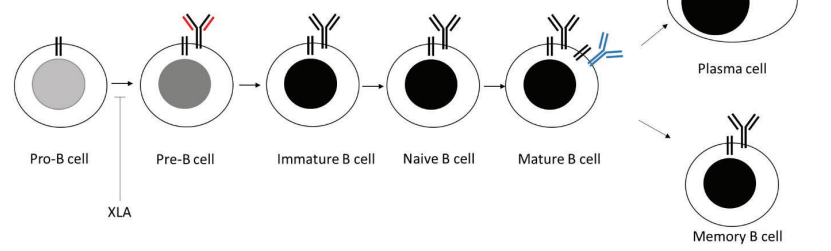

Figure 2. The different stages of B cell development.

The B cell development is governed by the pre-B cell receptor (pre-BCR) and B-cell receptor (BCR) which are formed by surface immunoglobulins and other cellular proteins. The signalling event is triggered when antigen binds to the membrane immunoglobulin; the receptors oligomerized and trigger downstream signalling pathways for cell growth, differentiation, adhesion and migration. This promotes SFKs such as Lyn and Lck to phosphorylate immunoreceptor tyrosine-based activation motive (ITAM) on both $\operatorname{Ig} \alpha$ and $\operatorname{Ig} \beta$. The phosphorylated ITAM motifs act as a docking site for SFKs and Syk ${ }^{[50,51]}$. The binding activates SFKs and Syk. The activated SFKs and Syk then phosphorylate and activate phosphoinositide 3-kinase (PI3K), resulting in the conversion of phosphatidylinositol-4,5-bisphosphate ( $\mathrm{PIP}_{2}$ ) to phosphatidylinositol-3,4,5-triphosphate $\left(\mathrm{PIP}_{3}\right)^{[52]}$. The resultant $\mathrm{PIP}_{3}$ formed on the membrane recruits Btk to the plasma membrane via interaction between PIP $_{3}$ and $\mathrm{PH}$ domain of $\mathrm{Btk}^{[23,50,53]}$. Syk also phosphorylates BLNK to create a binding site for specific $\mathrm{SH} 2$-domain containing proteins, including Btk and PLC $\gamma^{[54]}$. The binding of Btk to BLNK brings Btk in close proximity to Syk and SFKs, allowing them to transphosphorylate Btk at Tyr551 ${ }^{[38]}$. As discussed earlier, phosphorylation of Tyr551 facilitates Btk to further autophosphorylate itself on Tyr223. Phosphorylation at Tyr223 prevents the binding of inhibitory protein to Btk, enabling Btk to interact with its substrates ${ }^{[17]}$. The fully activated Btk can then phosphorylates and activates PLC $\gamma$ which cleaves $\mathrm{PIP}_{2}$ to inositol-1,4,5-trisphosphate (IP3) and diacylglycerol (DAG). IP3 and DAG are secondary messengers that direct calcium mobilization and activation of mitogen-activated protein kinase (MAPK) pathway through protein kinase $\mathrm{C}(\mathrm{PKC})$ and nuclear factor kappa-lightchain-enhancer of Activated B cells (NF- $\mathrm{B}$ ) pathway (Figure 3) ${ }^{[55,56]}$.

Ever since Btk was found highly expressed in B cells and constitutive activation of Btk causes sustained signalling which contribute to prolonged cell survival and over-proliferation of malignant B cells, thus, Btk has become the therapeutic target for B cell malignancies.

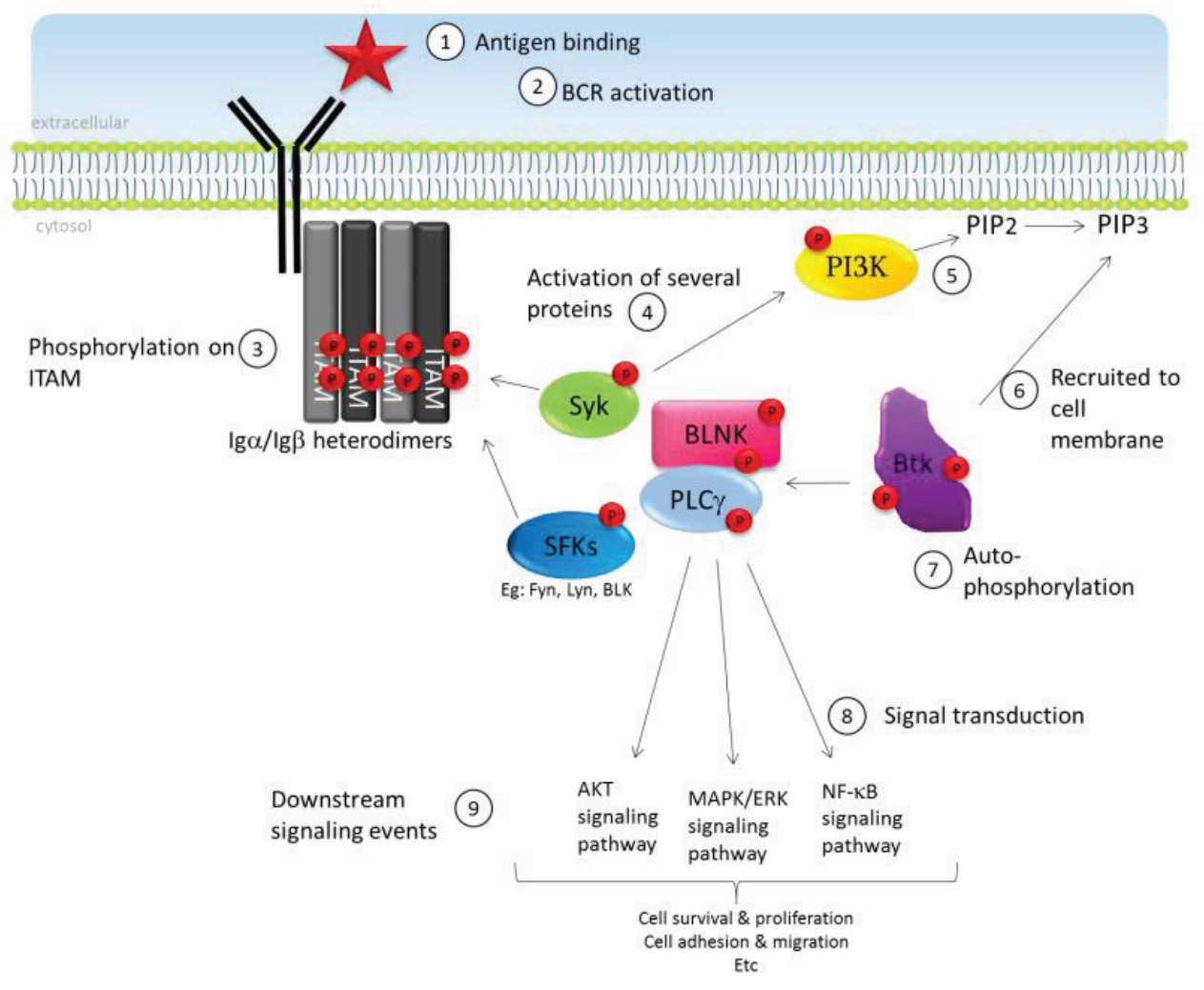

Figure 3. Summary of early events of BCR signalling pathways and the consequences after Btk's activation. 
THE BRUTON'S TYROSINE KINASE SPECIFIC INHIBITOR, IBRUTINIB

\section{Ibrutinib - the treatment of B cell malignancies}

Ibrutinib is a specific Btk inhibitor, mainly used for the treatment of Mantle Cell Lymphoma (MCL) and Chronic Lymphocytic Leukemia (CLL) as approved in the year 2013 and 2014 respectively by Food and Drug Administration $(\mathrm{FDA})^{[57]}$. This approval was driven by the promising results demonstrated in the phase 2 clinical trial that involved a total of 111 patients with relapsed or refractory $\mathrm{MCL}^{[57,58]}$. These patients, either with or without treatment of the therapeutic proteasome inhibitor, bortezomib (another type of chemotherapy drug for B cell malignancies) before, were treated with oral $560 \mathrm{mg}$ per day of ibrutinib until undesirable events had occurred. The results revealed $68 \%$ of high response rate and $58 \%$ of estimated overall survival rate at 18 months. Moreover, ibrutinib was found to be well-tolerated without critical toxic effects ${ }^{[58]}$. This outcome had eventually led to the clinical trials of ibrutinib in relapsed CLL. The results revealed that the drug improved the chance of remissions in patients with $75 \%$ of progression-free survival rate and $83 \%$ of overall survival rate $^{[59]}$.

\section{Development and design of ibrutinib as a highly selective Btk inhibitor}

Ibrutinib (or Imbruvica, formerly referred as PCI-32765) was one of the several irreversible inhibitors designed by Pan, Z. et. al. (2007) ${ }^{[60]}$ at Celera Genomics for the Celera's small molecule Btk inhibitory discovery program. A series of irreversible inhibitors was generated during the program, with the focus to target Btk and specifically inhibit its kinase activity. The compounds developed in the discovery program were later acquired by Pharmacyclics and Janssen, followed by clinical trials of ibrutinib for various haematological malignancies ${ }^{[57]}$.

During the drug development, inhibitors were de- signed to target the following structural features of Btk in order to have high selectivity against Btk $^{[60]}$ : (i) ATP binding cleft of Btk kinase domain in the inactive form (ii) nucleophilic amino acid residue unique to Btk kinase domain and (iii) the gatekeeper residue governing the accessibility of the active site to $\mathrm{Mg}^{2+}-\mathrm{ATP} / \mathrm{Mn}^{2+}-\mathrm{ATP}$.

For inhibiting kinases, ATP binding cleft (active site) in the kinase domain is a common targeted site. This is to block the binding of ATP and inhibits activity of the kinase ${ }^{[6]]}$. The inactive kinase domain is preferably targeted than active kinase domain. This is because active kinases share similar catalytic structure, but the inactive kinase domains adopt distinct conformation ${ }^{[62]}$. Despite that, this strategy alone is not enough to provide high selectivity of the drug as the active site is still highly conserved among many kinases ${ }^{[61,63]}$. Therefore, Pan et. al. (2007) ${ }^{[60]}$ focused on developing inhibitor with an electrophilic functional moiety capable of forming a covalent bond with Btk to increase selectivity of the inhibitor.

In order to increase the selectivity, the authors screened for nucleophilic amino acid residues that are unique to Btk $^{[60]}$. Cysteine 481 (Cys481) that resides in ATP binding pocket of Btk was postulated to be the suitable nucleophilic site that can covalently interact with the electrophilic inhibitors ${ }^{[00]}$. Sequence alignments of the sequences of 491 protein kinases had shown that only Btk and nine other protein kinases shared a cysteine residue at a homologous position in the kinase domain. The other nine kinases were: Blk, Bmk, EGFR, ErbB2, ErbB4, Itk, Jak3, Tec and Txk as shown in Figure $4^{[60,61]}$. Among them, Itk, Tec and Txk are members of the Tec family tyrosine kinase. This had provided a good strategy to greatly increase the selectivity of the inhibitor. Thus, a small molecule inhibitor with an enamide double bond group that could form covalent bond with the thiol group (-SH) of Cys481 was created.

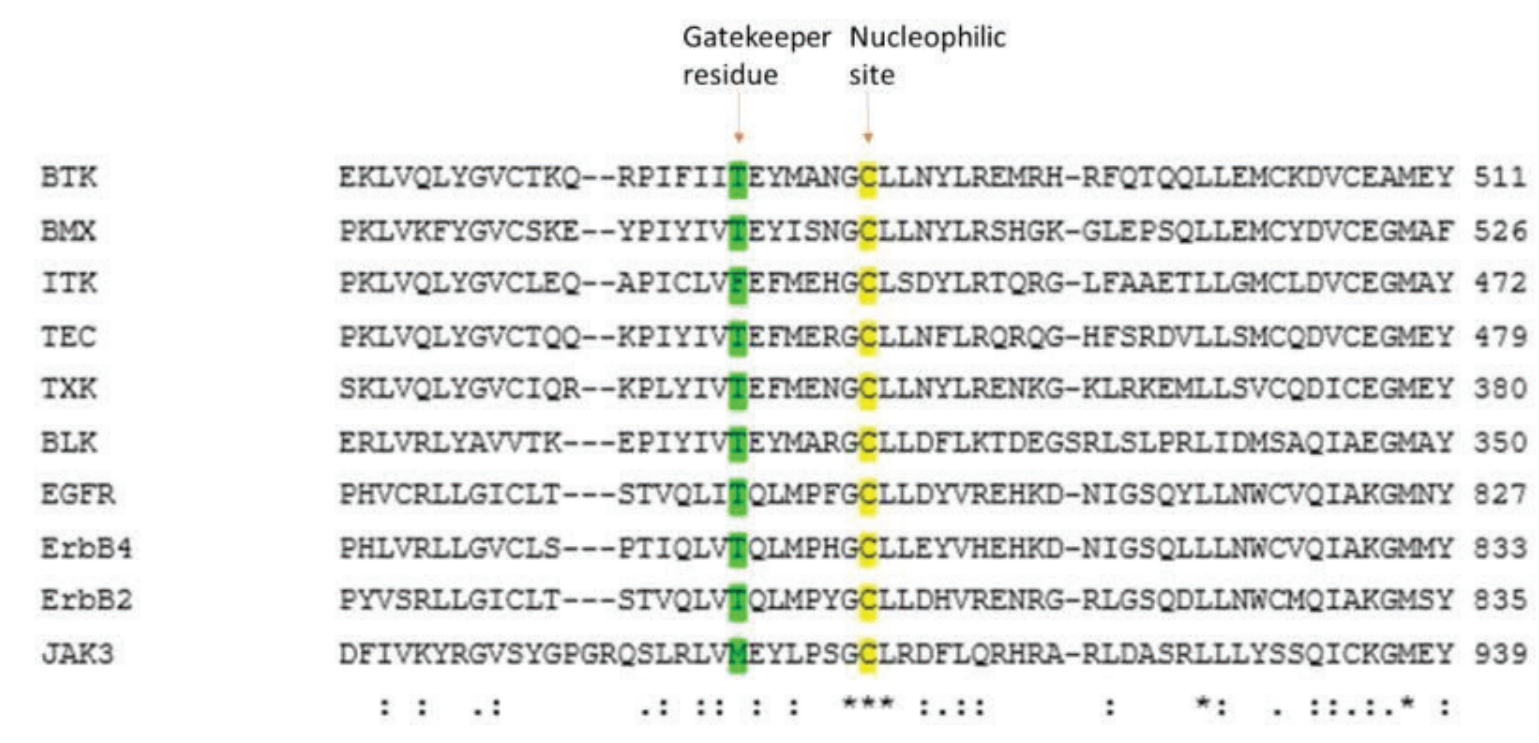

Figure 4. Sequence alignment of the 10 protein kinases that consisted cysteine residue at position same as Cys 481 of Btk. Protein sequences of the 10 protein kinases were obtained from UniprotKB and aligned using ClustalW2. Only the fragment of the multiple sequence alignment that contains the nucleophilic site (cys) and gatekeeper residue is shown here. Btk's Cys 481 is highlighted as yellow and Thr 474 as green. The cysteine and gatekeeper residues of the other 9 protein kinases were also highlighted as yellow and green respectively. All of them contained Cys residue at the same position as Cys 481 of Btk, and Thr residue as the gatekeeper residue except Itk and Jak3. 
Besides Cys481, the gatekeeper residue was also considered in the design of Btk inhibitors. This special residue plays an important role as selectivity filter of kinase inhibitors. Gatekeeper residue is the amino acid residue that lies behind the ATP binding site ${ }^{[61,63]}$. In some of the protein kinases, the gatekeeper residue contains a bulky aromatic side chain restricting the accessibility of the active site to compounds with bulky moiety - Ibrutinib is one of these compounds ${ }^{[63]}$. In Btk, the gatekeeper residue threonine 474 ( $\mathrm{Thr} 474)$ consists of relatively small hydroxyl $(-\mathrm{OH})$ and methyl groups $(-\mathrm{CH} 3)$ in its side chain. Only $20 \%$ of protein kinases in the kinome contain threonine as the gatekeeper residue. This approach can potentially exclude $80 \%$ of protein kinases in the kinome as targets of ibrutinib and further increases the selectivity of ibrutinib as Btk inhibitor ${ }^{[63]}$.

A series of Btk's irreversible inhibitors had been synthesized, followed by screening of selectivity and efficacy of the generated inhibitors in inhibiting a list of protein kinases' activity (including Btk). Ibrutinib possesses highest selectivity against Btk with $\mathrm{IC}_{50}$ of $0.5 \mathrm{nM}$ (in vitro) ${ }^{[64]}$ and only a few of protein kinase could be derivatized by ibrutinib at low concentration. For instance, it was reported that ibrutinib had $\mathrm{IC}_{50}$ of $1 \mathrm{nM}$ for Blk, Bmk and Tec while it had higher value of $\mathrm{IC}_{50}$ for the other suspected protein kinase (EGFR, Itk and Jak3) that is able to be bound by ibrutinib $^{[63]}$. As a result, ibrutinib (PCI-32765) was selected and proceeded into clinical studies. The promising outcome of the clinical trials had enabled ibrutinib to be approved as drug for treatment of MCL and CLL.

\section{BTK'S PATHOLOGICAL ROLE IN MULTIPLE MYELOMA (MM)}

Owing to the remarkable result of using ibrutinib for the treatment of MCL and CLL, several studies have started to explore its efficacy for the treatment of other types of B cell malignancies such as MM. As suggested earlier, Btk is expressed in hematopoietic cells from B cell lineage and repressed in the terminally differentiated plasma cell ${ }^{[65,66]}$. However, it has been demonstrated that Btk expression is elevated in MM cell lines and also in cancer cells obtained from MM patients ${ }^{[1,6,12]}$.

Besides, Btk was able to amplify its gene expression by activating $\mathrm{NF}-\kappa \mathrm{B}^{[55,67]}$. The activated $\mathrm{NF}-\kappa \mathrm{B}$ then binds and activates transcription of Btk gene ${ }^{[55,67]}$. The activation of $\mathrm{NF}-\kappa \mathrm{B}$ requires active Btk and PLC $\gamma^{[55,56]}$. The signalling pathways are not clear but Btk was found to be involved in $\mathrm{B}$ cell activating factor (BAFF) mediated activation of $\mathrm{NF}-\kappa \mathrm{B}^{[56,68]}$. In addition, the expression of Btk was significantly higher in dexamethasone- and also bortezomibresistant MM patients and cell lines, supporting the fact that increase in Btk expression is correlated with increasing activity of NF- $\kappa \mathrm{B}^{[69,70]}$. These findings may have suggested that malignant plasma cells could potentially utilise the upregulated Btk as an alternative mechanism to promote cell growth and survival, contributing to chemoresistance.

Moreover, Btk participates in maturation and differentiation of osteoclast (OC) in bone marrow ${ }^{[71,72]}$. OC is a type of bone cell in which its bone resorption function is upregulated in MM and contributes to the MM-related osteolysis. The function of Btk in MM was also evaluated in a study conducted by Tai et al. (2012) $)^{[12]}$ through Btk knockdown and the findings of this study have confirmed the pathogenic role of Btk activation in promoting MM cell growth and survival, interaction with other bone marrow stromal components, and inducing MMrelated osteolytic bone disease. Alternatively, inhibition of endogenous Btk was found to negatively impact on myeloma cell growth, migration, and adhesion to microenvironment ${ }^{[1,12,73]}$. The inhibition was reported to induce caspase-dependent cell death, which overruled Btk's role in inhibiting Fas-mediated apoptosis as mentioned earlier ${ }^{[6]}$. Overall, there is increasing evidence on the involvement of Btk in regulating MM cell growth, survival, migration and adhesion. Therefore, ibrutinib can potentially be use for the treatment of MM or relapsed MM.

\section{IBRUTINIB FOR MULTIPLE MYELOMA (MM)}

Given that many studies have suggested the involvement of Btk in several signalling pathways that are related to cell survival and proliferation, as well as cell adhesion and migration in MM, thus, researchers are motivated to trial the drug as a therapeutic option for MM. It has been demonstrated that ibrutinib is cytotoxic to malignant plasma cells through in vitro experiments. According to Cell Titer GLO assay conducted by Rushworth et al. $(2013)^{[6]}$, ibrutinib $(10 \mu \mathrm{M})$ had induced significant plasma cell death of about $7 \%-46 \%$ in MM cells. Besides, the cytotoxicity against malignant plasma cells in MM was significantly increased when ibrutinib was utilized in combination with either bortezomib or lenalidomide, thus, suggesting the synergistic effect of ibrutinib with these drugs ${ }^{[6]}$.

Apart from in vitro experimentation, several clinical trials have been conducted to examine the efficacy of ibrutinib for treatment of MM, either as monotherapy or in combination with other drugs. For example, a phase 2 open-label dose escalation trial conducted by Vij et al. (2014) ${ }^{[74]}$ involving the use of ibrutinib as a single agent monotherapy or in combination therapy with dexamethasone was examined in patients with relapsed and relapsed/refractory MM. The preliminary results indicated that both treatments: ibrutinib as single agent and ibrutinib in combination with dexamethasone, have produced anti-tumor effect in the patients. It was also reported that there was a trend towards enhanced efficacy in treatment with $840 \mathrm{mg}$ ibrutinib (daily) and $40 \mathrm{mg}$ dexamethasone (weekly) with well tolerated and manageable toxicities.

Additionally, the treatment using ibrutinib in combination with carfilzomib \pm dexamethasone was first evaluated in patients with relapsed or relapsed/refractory MM by Chari et al. (2018) ${ }^{[75]}$ in a phase 1 dose-finding trial. The outcomes demonstrated the combination therapy of ibrutinib $(840 \mathrm{mg})$ and carfilzomib $\left(36 \mathrm{mg} / \mathrm{m}^{2}\right)$ with dexamethasone showed the most promising result as it has the shortest median duration of response for patients who achieved above partial response which was 7.2 months. Hence, this dosage was recommended for the following phase 2 trial. 


\section{FUTURE DIRECTIONS AND CONCLUSION}

In light of the recent advances in the use of ibrutinib for treatment of MM, it is therefore important to chart the Btk oncogenic signalling pathway in MM cells. Other than the involvement of Btk in regulating NF- $\mathrm{BB}$ and stromal cell-derived factor-1 (SDF-1) induced signalling pathways $^{[1,12,76]}$, not much is known about its actual role in MM cells.

In myeloma cells, ibrutinib activity seems to be mediated through interfering NF- $\mathrm{KB}$ signalling pathway which subsequently promoting cell apoptosis ${ }^{[77]}$. Several studies also proposed that the immunomodulatory effects of Btk inhibitors on macrophages in tumor microenvironment and direct cytotoxic effects of Btk inhibitors on malignant B cells may be contributing to the therapeutic efficacy of these medicines ${ }^{[78]}$. Apparently, combination therapy of ibrutinib with other drugs is more effective than monotherapy for the treatment of MM. Furthermore, ibrutinib is still more effective in treating CLL than MM. This could be due to the reason that the relative protein expression of Btk in MM cells is significantly lower than that in CLL cells ${ }^{[6]}$, hence, MM cells are less sensitive towards ibrutinib compared to CLL cells. Also, it is possible that MM cell survival and proliferation may be supported by other complementary signalling pathways which are yet to be determined in the future. Thus, further studies should be conducted to investigate the downstream Btk targets which can be utilized to improve the efficacy of ibrutinib and the overall treatment of MM.

\section{Authors Contributions}

The literature review and manuscript writing were performed by S-TB, LN-SL, and JW-FL.

\section{Conflict of Interest}

The authors declare that the research was conducted in the absence of any commercial or financial relationships that could be construed as a potential conflict of interest.

\section{Reference}

1. Bam R, Ling W, Khan S, et al. Role of Bruton's tyrosine kinase in myeloma cell migration and induction of bone disease. Am J Hematol 2013; 88(6): 463-471.

2. Middela S and Kanse P. Nonsecretory multiple myeloma. Indian J Orthop 2009; 43(4): 408-411.

3. Palumbo A and Anderson K. Multiple myeloma. N Engl J Med 2011; 364(11): 1046-1060.

4. Mehta GR, Suhail F, Haddad, RY, et al. Multiple myeloma. Disease-amonth: DM 2014; 60(10): 483-488

5. Dong H, Chen L, Chen X, et al. Dysregulation of unfolded protein response partially underlies proapoptotic activity of bortezomib in multiple myeloma cells. Leukemia \& Lrymphoma 2009; 50(6): 974-984.

6. Rushworth SA, Bowles KM, Barrera LN, et al. BTK inhibitor ibrutinib is cytotoxic to myeloma and potently enhances bortezomib and lenalidomide activities through NF-אB. Cell Signal 2013; 25(1): 106-112.

7. Richardson PG, Weller E, Lonial S, et al. Lenalidomide, bortezomib, and dexamethasone combination therapy in patients with newly diagnosed multiple myeloma. Blood 2010; 116(5): 679-686.

8. Suzuki K. Current therapeutic strategy for multiple myeloma. Jap J Clin Oncol 2013; 43(2): 116-124.

9. American Cancer Society. Multiple Myeloma overview. 2014
Available on http://www.cancer.org/acs/groups/cid/documents/ webcontent $/ 003065$-pdf.pdf

10. Raab, MS, Podar, K, Breitkreutz, I, et al. Multiple myeloma. Lancet 2009; 374(9686): 324-339

11. Munshi, NC and Anderson, KC. New strategies in the treatment of multiple myeloma. Clin Cancer Res 2013; 19(13): 3337-3344.

12. Tai Y-T, Chang BY, Kong S-Y, et al. Bruton tyrosine kinase inhibition is a novel therapeutic strategy targeting tumor in the bone marrow microenvironment in multiple myeloma. Blood 2012; 120(9): 1877-1887.

13. Burger JA and Buggy JJ. Bruton tyrosine kinase inhibitor ibrutinib (PCI-32765). Leuk Lymphoma 2013; 54(11): 2385-2391.

14. Burger JA and Chiorazzi N. B cell receptor signaling in chronic lymphocytic leukemia. Trends Immunol 2013; 34(12): 592-601.

15. Tsukada S, Saffran DC, Rawlings DJ, et al. Deficient expression of a B cell cytoplasmic tyrosine kinase in human X-linked agammaglobulinemia. Cell 1993; 72(2): 279-290.

16. Mano $\mathrm{H}$. Tec family of protein-tyrosine kinases: An overview of their structure and function. Cytokine Growth Factor Rev 1999; 10(3-4): 267-280

17. Mohamed AJ, Yu L, Bäckesjö CM, et al. Bruton's tyrosine kinase (Btk): Function, regulation, and transformation with special emphasis on the PH domain. Immunol Rev 2009; 228(1): 58-73.

18. Bruton OC. Agammaglobulinemia. Pediatrics 1952; 9(6): 722-728.

19. Camcioğlu Y. Bruton's Disease. In: Immunopathology and Immunomodulation. K Metodiev, Croatia: InTech; 2015: 217-243.

20. Smith C. From identification of the BTK kinase to effective management of leukemia. Oncogene 2017; 36(15): 2045-2053.

21. Hagemann TL, Chen Y, Rosen FS, et al. Genomic organization of the Btk gene and exon scanning for mutations in patients with $\mathrm{X}$ linked agammaglobulinemia. Hum Mol Genet 1994; 3(10): 1743 1749 .

22. Smith CE, Bäckesjö C-M, Berglöf A, et al. X-linked agammaglobulinemia: lack of mature B lineage cells caused by mutations in the Btk kinase. In: Springer Semin Immunopathol, Springer; 1998: 369-381.

23. Li T, Tsukada S, Satterthwaite A, et al. Activation of Bruton's tyrosine kinase (BTK) by a point mutation in its pleckstrin homology (PH) domain. Immunity $1995 ; 2(5): 451-460$.

24. Salim K, Bottomley MJ, Querfurth E, et al. Distinct specificity in the recognition of phosphoinositides by the pleckstrin homology domains of dynamin and Bruton's tyrosine kinase. EMBO J 1996; 15(22): 6241-6250.

25. Rawlings DJ, Saffran DC, Tsukada S, et al. Mutation of unique region of Bruton's tyrosine kinase in immunodeficient XID mice. Science 1993; 261(5119): 358-361.

26. Mohamed A, Nore B, Christensson B, et al. Signalling of Bruton's tyrosine kinase, Btk. Scand J Immunol 1999; 49(2): 113-118.

27. Hyvönen M and Saraste M. Structure of the PH domain and Btk motif from Bruton's tyrosine kinase: Molecular explanations for Xlinked agammaglobulinaemia. EMBO J 1997; 16(12): 3396-3404.

28. Yao L, Kawakami Y, and Kawakami T. The pleckstrin homology domain of Bruton tyrosine kinase interacts with protein kinase $\mathrm{C}$. Proc Natl Acad Sci 1994; 91(19): 9175-9179.

29. Yao L, Suzuki H, Ozawa K, et al. Interactions between protein kinase $\mathrm{C}$ and pleckstrin homology domains Inhibition by phosphatidylinositol 4, 5-bisphosphate and phorbol 12-myristate 13-acetate. J Biol Chem 1997; 272(20): 13033-13039.

30. Tsukada S, Simon MI, Witte ON, et al. Binding of beta gamma subunits of heterotrimeric $\mathrm{G}$ proteins to the $\mathrm{PH}$ domain of Bruton tyrosine kinase. Proc Natl Acad Sci 1994; 91(23): 11256-11260.

31. Yang W and Desiderio S. BAP-135, a target for Bruton's tyrosine kinase in response to B cell receptor engagement. Proc Natl Acad Sci 1997; 94(2): 604-609.

32. Vassilev A, Ozer Z, Navara C, et al. Bruton's tyrosine kinase as an inhibitor of the Fas/CD95 death-inducing signaling complex. J Biol Chem 1999; 274(3): 1646-1656

33. Vihinen M, Nilsson L, and Smith CE. Tec homology (TH) adjacent to the PH domain. FEBS Lett 1994; 350(2-3): 263-265.

34. Vihinen M, Nore BF, Mattsson PT, et al. Missense mutations affecting a conserved cysteine pair in the TH domain of Btk. FEBS Lett 1997; 413(2): 205-210.

35. Hansson H, Okoh MP, Smith CE, et al. Intermolecular interaction between the SH3 domain and the prolinerich TH region of Bruton's tyrosine kinase. FEBS Lett 2001; 489(1): 67-70.

36. Okoh MP and Vihinen M. Interaction between Btk TH and SH3 domain. Biopolymers 2002; 63(5): 325-334.

37. Gustafsson MO, Hussain A, Mohammad DK, et al. Regulation of nucleocytoplasmic shuttling of Bruton's tyrosine kinase (Btk) through a novel SH3-dependent interaction with ankyrin repeat domain 54 (ANKRD54). Mol Cell Biol 2012; 32(13): 2440-2453.

38. Tzeng SR, Pai MT, Lung FDT, et al. Stability and peptide binding specificity of Btk SH2 domain: Molecular basis for X-linked agammaglobulinemia. Protein Sci 2000; 9(12): 2377-2385.

39. Baba Y, Hashimoto S, Matsushita M et al. BLNK mediates Sykdependent Btk activation. Proc Natl Acad Sci 2001; 98(5): 2582 2586.

40. Hashimoto S, Iwamatsu A, Ishiai M, et al. Identification of the SH2 domain binding protein of Bruton's tyrosine kinase as BLNKfunctional significance of Btk-SH2 domain in B-cell antigen receptor-coupled calcium signaling. Blood 1999; 94(7): 2357-2364.

41. Vetrie D, Vořechovský I, Sideras $\mathrm{P}$, et al. The gene involved in $\mathrm{X}$-linked agammaglobulinaemia is a member of the src family of protein-tyrosine kinases. Nature 1993; 361(6409): 226-233.

42. Dinh M, Grunberger D, Ho H, et al. Activation mechanism and 
steady state kinetics of Bruton's tyrosine kinase. J Biol Chem 2007; 282(12): 8768-8776.

43. Mahajan S, Fargnoli J, Burkhardt AL, et al. Src family protein tyrosine kinases induce autoactivation of Bruton's tyrosine kinase. Mol Cell Biol 1995; 15(10): 5304-5311.

44. Márquez JA, Smith CE, Petoukhov MV et al. Conformation of full length Bruton tyrosine kinase (Btk) from synchrotron X-ray solution scattering. EMBO J 2003; 22(18): 4616-4624.

45. $\mathrm{Xu} \mathrm{W}$, Harrison SC, and Eck MJ. Three-dimensional structure of the tyrosine kinase c-Src. Nature 1997; 385(6617): 595-602.

46. Park H, Wahl MI, Afar DE, et al. Regulation of Btk function by a majo autophosphorylation site within the SH3 domain. Immunity 1996; 4(5): 515-525.

47. Lowry WE, Huang J, Lei M, et al. Role of the PHTH module in protein substrate recognition by Bruton's agammaglobulinemia tyrosine kinase. J Biol Chem 2001; 276(48): 45276-45281.

48. Janeway CA, Travers P, Walport M, et al. Immunobiology: The Immune System in Health and Disease. Vol. 7. 2001, London: Current Biology

49. Khan WN. Regulation of B lymphocyte development and activation by Bruton's tyrosine kinase. Immunol Res 2001; 23(2-3): 147-156.

50. Rawlings DJ, Scharenberg AM, Park H, et al. Activation of BTK by a phosphorylation mechanism initiated by SRC family kinases. Science 1996; 271(5250): 822-825.

51. Monroe JG. ITAM-mediated tonic signalling through pre-BCR and BCR complexes. Nature Rev Immuno 2006; 6(4): 283-294.

52. Li Z, Wahl MI, Eguinoa A, et al. Phosphatidylinositol 3-kinase- $\gamma$ activates Bruton's tyrosine kinase in concert with Src family kinases. Proc Natl Acad Sci 1997; 94(25): 13820-13825.

53. Brown JR. Ibrutinib (PCI-32765), the first BTK (Bruton's tyrosine kinase) inhibitor in clinical trials. Curr Hematol Malig R 2013; 8(1): 1-6. Kurosaki T and Tsukada S. BLNK: Connecting Syk and Btk to calcium signals. Immunity $2000 ; 12(1): 1-5$.

55. Yu L, Mohamed AJ, Simonson OE et al. Proteasome-dependent autoregulation of Bruton tyrosine kinase (Btk) promoter via NF- $\mathrm{KB}$. Blood 2008; 111(9): 4617-4626.

56. Shinners NP, Carlesso G, Castro I, et al. Bruton's tyrosine kinase mediates NF- $\mathrm{KB}$ activation and $\mathrm{B}$ cell survival by $\mathrm{B}$ cell-activating factor receptor of the TNF-R family. J Immunol 2007; 179(6): 3872-3880.

57. Cameron F and Sanford M. Ibrutinib: First global approval. Drugs 2014; 74(2): 263-271.

58. Wang ML, Rule S, Martin P, et al. Targeting BTK with ibrutinib in relapsed or refractory mantle-cell lymphoma. New Engl J Med 2013; 369(6): 507-516

59. Byrd JC, Furman RR, Coutre SE, et al. Targeting BTK with ibrutinib in relapsed chronic lymphocytic leukemia. New Engl J Med 2013; 369(1): 32-42.

60. Pan Z, Scheerens H, Li SJ, et al. Discovery of selective irreversible inhibitors for Bruton's tyrosine kinase. Chem Med Chem 2007; 2(1): $58-61$

61. Lou Y, Owens TD, Kuglstatter A, et al. Bruton's tyrosine kinase inhibitors: Approaches to potent and selective inhibition, preclinical and clinical evaluation for inflammatory diseases and $\mathrm{B}$ cell malignancies. J Med Chem 2012 ; 55(10): 4539-4550.

62. Huse M and Kuriyan J. The conformational plasticity of protein ki- nases. Cell 2002; 109(3): 275-282.

63. Cohen MS, Zhang $C$, Shokat KM et al. Structural bioinformaticsbased design of selective, irreversible kinase inhibitors. Science 2005; 308(5726): 1318-1321

64. Honigberg LA, Smith AM, Sirisawad M, et al. The Bruton tyrosine kinase inhibitor PCI-32765 blocks B-cell activation and is efficacious in models of autoimmune disease and B-cell malignancy. Proc Natl Acad Sci 2010; 107(29): 13075-13080

65. de Weers M, Verschuren MC, Kraakman ME, et al. The Bruton's tyrosine kinase gene is expressed throughout B cell differentiation, from early precursor B cell stages preceding immunoglobulin gene rearrangement up to mature B cell stages. Eur J Immunol 1993; 23(12): 3109-3114

66. Smith C, Baskin B, Humire-Greiff P, et al. Expression of Bruton's agammaglobulinemia tyrosine kinase gene, BTK, is selectively down-regulated in T lymphocytes and plasma cells. J Immunol 1994; 152(2): 557-565.

67. Bajpai UD, Zhang K, Teutsch M, et al. Bruton's tyrosine kinase links the B cell receptor to nuclear factor $\kappa \mathrm{B}$ activation. J Exp Med 2000; 191(10): 1735-1744

68. Siebenlist U, Brown K, and Claudio E. Control of lymphocyte de-

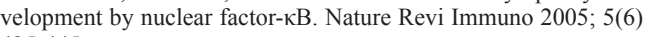
435-445.

69. Murray MY, Zaitseva L, Auger MJ, et al. Ibrutinib inhibits BTK driven NF- $\mathrm{KB}$ p65 activity to overcome bortezomib-resistance in multiple myeloma. Cell Cycle 2015; 14(14): 2367-2375.

70. Liu Y, Dong Y, Jiang QL, et al. Bruton's tyrosine kinase: potential target in human multiple myeloma. Leuk Lymphoma 2014; 55(1): 177-181.

71. Shinohara M, Koga T, Okamoto K, et al. Tyrosine kinases Btk and Tec regulate osteoclast differentiation by linking RANK and ITAM signals. Cell 2008; 132(5): 794-806.

72. Lee SH, Kim T, Jeong D, et al. The tec family tyrosine kinase Btk Regulates RANKL-induced osteoclast maturation. J Biol Chem 2008; 283(17): 11526-11534

73. Bam R, Venkateshaiah S, Khan S, et al. Role of Bruton's tyrosine kinase (BTK) in growth and metastasis of INA6 myeloma cells. Blood Cancer J 2014; 4(8): e234.

74. Vij R, Huff CA, Bensinger WI, et al. Ibrutinib, single agent or in combination with dexamethasone, in patients with relapsed or relapsed/refractory multiple myeloma (MM): Preliminary phase 2 results. Blood 2014; 124: 31

75. Chari A, Larson S, Holkova B, et al. Phase 1 trial of ibrutinib and carfilzomib combination therapy for relapsed or relapsed and refractory multiple myeloma. Leuk Lymphoma 2018; 59(11): 25882594

76. Tai YT and Anderson KC. Bruton's tyrosine kinase: oncotarget in myeloma. Oncotarget 2012; 3(9): 913-914.

77. Naymagon L and Abdul-Hay M. Novel agents in the treatment of multiple myeloma: a review about the future. J Hematol Oncol 2016; 9(1): 52 .

78. Ping L, Ding N, Shi Y, et al. The Bruton's tyrosine kinase inhibitor ibrutinib exerts immunomodulatory effects through regulation of tumor-infiltrating macrophages. Oncotarget 2017; 8(24): 3921839229. 